\title{
Construção de uma escala para medir o nível dos colaboradores para a prática da Melhoria Contínua em uma organização por meio do modelo de resposta nominal da TRI
}

\author{
Diego Milnitz ${ }^{\mathrm{a} *}$, Dalvio Ferrari Tubino ${ }^{\mathrm{a} * *}$ \\ aUniversidade Federal de Santa Catarina, Florianópolis, SC, Brasil \\ *dmilnitz@bol.com.br, **dtubino@uol.com.br
}

\begin{abstract}
Resumo
Esta pesquisa oferece uma discussão sobre a construção de uma escala para medir o nível dos colaboradores para a prática da Melhoria Contínua dentro de uma organização. 0 desenvolvimento desta foi possível por meio da utilização de um instrumento de medida constituído de 34 itens embasados no conceito de Melhoria Contínua e aplicados em uma amostra de 519 colaboradores pertencentes a uma organização industrial. A seguir os dados foram avaliados estatisticamente pela Teoria de Resposta ao ltem (TRI), mais precisamente por meio do modelo de resposta nominal. Como resultado, conseguiu-se a equalização entre os itens aplicados e os scores dos colaboradores pesquisados, permitindo o desenvolvimento de uma métrica com distintos níveis para a prática da Melhoria Contínua. As decorrências estratégicas derivadas deste resultado são muito importantes, pois na medida do traço latente a aplicação da TRl permitiu fazer uma avaliação do fenômeno em estudo com mais precisão e consistência, fornecendo assim, informações relevantes para a tomada de decisões dentro da organização pesquisa.
\end{abstract}

Palavras-chave

Melhoria contínua. Organização industrial. Métrica.

\section{Introdução}

0 cuidado com as competências existentes na organização pode garantir que elas sejam vistas em sua totalidade e, se utilizadas na prática da melhoria contínua, levam ao aperfeiçoamento autossustentado e continuado dos processos e produtos. Essa melhoria deve ser tratada de forma completa, o que requer equilíbrio e integração dos princípios técnicos e sociais. lsso conduz à necessidade de atuação em diferentes áreas e à consideração de aspectos como habilidades e motivação das pessoas (Harrison, 2000); isso pode ser constatado em outras pesquisas como as de Brannen et al. (1998) e de Harrison \& Storey (1996).

Chaves (2005) coloca em sua obra que o fator humano é imprescindível para que a prática da Melhoria Contínua aconteça, ou seja, é um sistema que visa promover o trabalho em equipe e o crescimento das pessoas por meio de uma constante troca de ideias e conhecimentos. Entretanto, muitas organizações têm aplicado esses conceitos de forma equivocada, sem levar em consideração a relevância das pessoas no processo, gerando muita frustração e descontentamento por parte dos tomadores de decisões, operadores e gestores de processo (Caffyn, 1999).

Para envolver as pessoas na Melhoria Contínua, as organizações precisam conhecer o nível dos colaboradores para a prática, possibilitando, deste modo, que estratégias possam ser desenvolvidas e implantadas com o intuito de facilitar o envolvimento das pessoas com a aplicação desses princípios. lsso possibilita que as organizações reduzam o índice de insucesso neste processo. Porém, a maioria das pesquisas nessa área busca compreender e quantificar 
o nível das organizações para a prática da Melhoria Contínua, considerando as pessoas somente como uma parte do processo. lsso pode ser observado nos trabalhos de Caffyn (1999), Bessant et al. (2001) e Mesquita \& Alliprandini (2003), entre outros.

Dada a importância que as pessoas têm com o melhoramento contínuo dos processos e produtos e numa tentativa de contribuir para essa lacuna de pesquisa, o presente artigo propõe construir uma escala para medir o nível dos colaboradores para a prática da Melhoria Contínua em uma organização industrial. Essa medida possibilitará aos interessados entender em qual nível cada colaborador se localiza quanto a essa prática, dando subsídios para que sejam desenvolvidos planejamentos adequados e consequentemente gere maior assertividade, agilidade, eficiência e menos custos com a implementação das práticas de Melhoria Contínua.

Além dessa introdução, contextualizando o objetivo da pesquisa, o artigo apresenta alguns conceitos sobre Melhoria Contínua, seção 2. Em seguida, na Seção 3, é apresentado o procedimento metodológico aplicado, seguido por um subitem que explica a aplicação do Modelo de Resposta Nominal. Na seção 4, o método é aplicado e, consequentemente, na seção 5, é realizada a construção da escala de medida. Finalmente, na seção 6, são realizadas algumas considerações finais do trabalho com sugestões para futuras pesquisas.

\section{Definindo Melhoria Contínua (MC)}

Segundo Jha et al. (1995) e Bhuiyan \& Baghel (2005), a aplicação do conceito de Melhoria Contínua (MC) teve início no século XIX com o advento da administração científica. Tendo seu auge nas práticas de melhorias adotadas pelas empresas japonesas após a Segunda Guerra Mundial (Ritzman \& Krajewski, 2004). Esse método está embasado na crença de que o sistema produtivo pode ser continuamente melhorado, dessa forma, nenhum dia pode passar sem que a empresa melhore sua posição competitiva (Martins \& Laugeni, 2006).

Para Cole (2001), o processo de MC é construído por meio do aprendizado que tem um caráter investigativo como característica fundamental, sendo que sua essência está na capacidade de solucionar problemas de modo efetivo. Além disso, é necessário que a cultura e a infraestrutura da organização proporcionem a melhoria contínua bem como os métodos e ferramentas que promovam a sua implantação (Bessant et al., 1994).

Neste sentido, o fator humano se torna essencial, ou seja, é um sistema que visa promover o trabalho em equipe e o crescimento das pessoas por meio de uma constante troca de ideias e conhecimentos (Chaves,
2005). De forma similar Shiba (1997), afirma que Melhoria Contínua significa o envolvimento de todos os colaboradores da empresa no sentido de buscar de forma constante e sistemática o aperfeiçoamento dos produtos e processos empresariais.

Além disso, alguns autores, como Recht \& Wilderom (1998) e Bessant et al. (1994), alegam que, por ser um conceito simples, de fácil entendimento e de baixo nível de investimento, a Melhoria Contínua tem sido amplamente aplicada como uma das formas mais eficientes de aumentar a competitividade nas fábricas. No entanto, existem muitas empresas que têm experiências frustradas em relação a sua implantação. Por exemplo, um grande número de empresas concentra-se exclusivamente na aplicação das ferramentas, esquecendo-se dos outros aspectos organizacionais como as pessoas ou a infraestrutura da organização (Bessant et al., 2001). Outras têm pressa em atingir os benefícios, não levando em conta que seu desenvolvimento requer tempo, dedicação e implica, primeiramente, em fazer tentativas e adotar uma consciência própria do novo jeito de se fazer as coisas (Caffyn, 1999).

Conforme Caffyn (1999), a falta de clareza sobre os conceitos da Melhoria Contínua pode levar as empresas a se equivocarem no que diz respeito a sua implantação. Assim, por meio de suas pesquisas, os autores conseguiram identificar as habilidades básicas e as normas comportamentais, constatando que nem todas as empresas que dizem praticar estes conceitos realizam uma gestão que considera aspectos relacionados ao desenvolvimento das habilidades dos colaboradores.

Na Tabela 1 são sintetizados os níveis de habilidades e comportamentos esperados para a prática da Melhoria Contínua (MC). A primeira coluna mostra os níveis da Melhoria Contínua que devem ser apresentados e relacionados a cada um dos níveis; na segunda coluna, estão descritos os padrões comportamentais. Eles representam os modelos de comportamento que devem estar presentes, a fim de se ter a prática da Melhoria Contínua. No entanto, a sistematização desse padrão serve somente como um mapa para identificar o nível para a prática da Melhoria Contínua e como uma forma de orientação para aperfeiçoar cada capacidade identificada (Bessant et al., 2001).

\section{Procedimentos metodológicos}

Este trabalho tem as características de uma pesquisa survey, tendo como objetivo ser um artigo de levantamento descritivo, realizado para entender a relevância de certo fenômeno e descrever a distribuição deste em uma população (Filippini, 1997). 
Tabela 1. Niveis para a prática da Melhoria Contínua nas organizações.

\begin{tabular}{|c|c|c|}
\hline & Níveis & Padrões de Comportamento (Atitudes) \\
\hline Nível 1 & $\begin{array}{l}\text { Pré-Melhoria Contínua: Existe um interesse inicial } \\
\text { pela prática motivado por uma crise, visita a } \\
\text { outra organização ou participação em algum } \\
\text { evento de melhoria; porém a prática é pontual. }\end{array}$ & $\begin{array}{l}\text { Os problemas são resolvidos aleatoriamente; não existe nenhum esforço } \\
\text { ou estrutura formal para melhorar; as ocorrências são ocasionais de } \\
\text { melhorias pontuadas por inatividade e não participação; as soluções } \\
\text { visam os benefícios de curto prazo; não há nenhum impacto estratégico } \\
\text { sobre os recursos humanos, financeiros ou outras metas mensuráveis. }\end{array}$ \\
\hline Nível 2 & $\begin{array}{l}\text { Melhoria Contínua estruturada: Existe um } \\
\text { envolvimento com a prática que objetiva disseminar } \\
\text { os conceitos da Melhoria Contínua por toda a } \\
\text { organização por meio de um método adequado. }\end{array}$ & $\begin{array}{l}\text { Uma iniciativa ou organização de melhoria contínua é introduzida; as } \\
\text { áreas de apoio usam processos estruturados de solução de problemas; } \\
\text { uma grande parte das áreas de apoio participa das atividades de melhoria } \\
\text { contínua; as áreas de apoio são treinadas nas ferramentas básicas de } \\
\text { melhoria contínua; existe a introdução de um sistema de gerenciamento } \\
\text { de ideias; e um sistema de reconhecimento e recompensas; as atividades de } \\
\text { melhoria contínua estão integradas nas operações do dia a dia. }\end{array}$ \\
\hline Nível 3 & $\begin{array}{l}\text { Melhoria Contínua orientada para metas: Há um } \\
\text { compromisso com a prática da Melhoria Contínua } \\
\text { e com os resultados estratégicos esperados pela } \\
\text { organização, ou seja, os colaboradores estão } \\
\text { preocupados com a estratégia e o impacto das } \\
\text { melhorias nos resultados. }\end{array}$ & $\begin{array}{l}\text { Todos os comportamentos do nível dois são considerados e também existe } \\
\text { o desdobramento formal dos objetivos estratégicos, monitoramento e } \\
\text { medição da melhoria contínua em relação aos objetivos, as atividades } \\
\text { de melhoria contínua fazem parte das atividades principais do negócio e } \\
\text { o foco inclui a solução de problemas entre áreas da empresa (processos } \\
\text { interorganizacionais) ou mesmo entre empresas (cadeias de produção). }\end{array}$ \\
\hline Nível 4 & $\begin{array}{l}\text { Melhoria Contínua Proativa: Os colaboradores têm } \\
\text { autonomia e poder para gerenciar e dirigir seus } \\
\text { próprios processos e suas iniciativas de melhorias. } \\
\text { Neste nível os colaboradores têm um senso de dono } \\
\text { do processo muito elevado. }\end{array}$ & $\begin{array}{l}\text { Todos os comportamentos dos níveis dois e três são considerados, } \\
\text { a melhoria contínua é desenvolvida e focada na solução proativa } \\
\text { de problemas, tendo altos níveis de práticas de experimentação e } \\
\text { aprendizagem. }\end{array}$ \\
\hline Nível 5 & $\begin{array}{l}\text { Plena capacidade de Melhoria Contínua: Neste } \\
\text { nível o colaborador além de praticar também exerce } \\
\text { o papel de instrutor para a prática da Melhoria } \\
\text { Contínua. }\end{array}$ & $\begin{array}{l}\text { Todos os comportamentos dos níveis dois, três e quatro são considerados, } \\
\text { sendo que o aprendizado organizacional ocorre de modo extensivo e } \\
\text { largamente distribuído, é comum a iniciativa para procura e solução } \\
\text { sistemática de problemas de forma mais proativa; além da aquisição e o } \\
\text { compartilhamento do conhecimento obtido, a experimentação ocorre de } \\
\text { forma abrangente e autônoma, mas, gerenciada. }\end{array}$ \\
\hline
\end{tabular}

Fonte: Adaptado de Bessant et al. (2001).

Sua finalidade primária não é o desenvolvimento de teorias, embora, diante dos fatos descritos, ele possa fornecer sugestões úteis para a construção da teoria e seu refinamento (Miguel et al., 2009). Ainda conforme Gil (1996), este pode ser considerado descritivo porque busca evidenciar as características de um determinado fenômeno, envolvendo o uso de técnicas padronizadas de coleta de dados e questionário com um conjunto de itens.

Este estudo aplica ambos os métodos quantitativos e qualitativos no seu desenvolvimento. É quantitativo quando garante a exatidão dos resultados impedindo distorções de análise e interpretação (Hernandez \& Caldas, 2001). Utilizam-se também os métodos quantitativos para a interpretação dos fenômenos e para a compreensão detalhada dos significados e características situacionais apresentadas pelos entrevistados (Richardson, 1989). Neste caso, os significados e características situacionais estão relacionados com os conceitos e as classificações, atribuídas ao princípio de Melhoria Contínua.

Este estudo proporciona ainda uma reflexão indutiva, utilizando um conjunto de variáveis secundárias para fazer inferência do traço latente que representa o objeto do estudo, tais como as conclusões obtidas nas análises dos parâmetros dos itens e dos níveis dos colaboradores para a prática da Melhoria Contínua que foram estimados do conjunto de itens.

\subsection{Modelo de resposta nominal da Teoria de Resposta ao Item}

Inseridos num contexto de mudanças e transformações técnicas, sociais e econômicas, enfatiza-se a importância de encontrar novas metodologias que proporcionem condições para que essas áreas se desenvolvam (Meijer, 2003). Neste sentido, a avaliação tem sido um instrumento que representa com maior eficácia essas transformações (Bortolotti et al., 2012).

Avaliar, no sentido de medir, abrange não somente a avaliação de desempenho, mas também medir a satisfação por um serviço, a preferência por determinado produto, etc. A satisfação sendo definida como uma condição psicológica não pode ser observada e nem medida diretamente, sendo, deste modo, considerada um traço latente (Bortolotti et al., 2012). Contudo, devido à necessidade de avaliar certas características, tem-se buscado desenvolver escalas para medi-las (Bortolotti et al., 2012).

Conforme Araujo et al. (2009), a Teoria de Resposta ao Item (TRI) compreende modelos para avaliar traços latentes. Estes modelos apresentam formas de representar a relação entre a probabilidade de um indivíduo dar uma resposta a um item e seus traços latentes ou habilidades, na área de conhecimento a ser avaliada ou verificada, os quais não podem ser observados diretamente. Bock (1972) desenvolveu 
um modelo baseado no modelo logístico de dois parâmetros que pode ser aplicado a todas as categorias de resposta escolhidas em um teste com itens de múltipla escolha.

Segundo Araujo et al. (2009), o propósito deste modelo de resposta nominal é maximizar a precisão da habilidade estimada usando toda a informação contida nas respostas dos indivíduos, e não apenas se o item é respondido corretamente ou não. De acordo com este modelo de natureza acumulativa, indivíduos com maior nível para a prática da Melhoria Contínua na organização têm maior probabilidade de alcançar a categoria superior em relação à resposta. Portanto, pelo uso desse modelo da TRl é possível construir uma escala de medida para representar o nível dos colaboradores para a prática da Melhoria Contínua nas organizações que possuam este processo. Na próxima seção, serão abordados aspectos sobre a aplicação desse método.

\section{Aplicação do método}

Para possibilitar a avaliação e construção de uma escala de medida que representasse o nível dos colaboradores para a prática da Melhoria Contínua na organização, foram usados 34 itens desenvolvidos e aplicados em trabalhos de Bessant et al. (2001), Mesquita \& Alliprandini (2003) e Oprime \& Lizarelli (2010), promovendo assim, o desenvolvimento de um instrumento de medida adequado para posterior aplicação e análise por meio da teoria de resposta ao item. Esses itens tem o objetivo de captar a percepção e o conhecimento dos colaboradores com relação à prática da Melhoria Contínua na organização pesquisada, possibilitando, dessa maneira, que seu traço latente possa ser medido.

$\mathrm{Na}$ Tabela 2, constam todos os itens que compõem o instrumento que foi respondido pelos colaboradores dessa organização. Esses itens foram adequados para permitir maior abrangência das cinco faixas dos níveis propostos por Bessant et al. (2001). Cada item contém duas categorias de resposta: Não (0) e Sim (1).

Depois da construção do instrumento contendo 34 itens, este foi aplicado em uma amostra de 519 colaboradores de uma organização industrial que já adotava a prática da Melhoria Contínua. Portanto, se tentou abranger dentro desse grupo colaboradores de todos os níveis da prática de Melhoria Contínua, segundo Bessant et al. (2001). Para construção da amostra, foi utilizado o método de amostragem por conveniência, já que para montar uma escala de medida é necessário que existam respondentes de todos os níveis avaliados (Bortolotti et al., 2012).
Para verificar a qualidade do conjunto de itens aplicado, foi utilizada a análise de dimensionalidade, por meio da extração das cargas do modelo de análise fatorial por componentes principais. Usando-se o software "R 2.13.2" para a verificação da unidimensionalidade do conjunto composto pelos 34 itens. Na Figura 1, é apresentado o gráfico com os componentes e seus respectivos autovalores. Nesta figura, observa-se que um fator se destaca em relação aos demais, indicando assim a unidimensionalidade do conjunto de itens avaliados.

Ainda segundo Reckase (1979), os resultados da análise fatorial podem indicar a unidimensionalidade, se o primeiro fator for maior ou igual a $20 \%$ da variância total dos componentes avaliados. Como se observa na Tabela 3, o primeiro fator explica 30\% da variância total, logo, é aceitável ponderar que o conjunto dos 34 itens tem um fator dominante.

Para estimação e posterior avaliação dos parâmetros dos itens e dos colaboradores, alguns procedimentos foram adotados. Primeiro as duas categorias de resposta do instrumento foram codificadas: $0=$ Não; e $1=\operatorname{Sim}$. As respostas dos 34 itens foram analisadas pelo modelo de resposta nominal, por meio do programa Multilog. As análises realizadas foram obtidas utilizando 19 pontos de quadratura, 100 ciclos externos, 28 ciclos de interação para os parâmetros dos itens e, como critério de convergência, utilizou-se o valor de 0,0001.

Na Tabela 4, constam os valores estimados dos parâmetros $\mathrm{a}^{0}, \mathrm{a}^{1}, \mathrm{c}^{0}, \mathrm{c}^{1}$. Como na aplicação do modelo de resposta nominal, não se considerou resposta certa ou errada. Para qualificar o item âncora ou quase âncora, foi utilizada a categoria $1=\operatorname{Sim}$, pois, como o objetivo da pesquisa era construir uma escala de medida do nível para a prática da Melhoria Contínua, este item demonstrou o quão predisposto à prática 0 colaborador estava. Sendo assim, a outra categoria não foi considerada na avaliação dos itens âncoras e quase âncoras.

Por essa tabela, também é possível avaliar a qualidade e a dificuldade de cada item. A qualidade é demonstrada pelo parâmetro "a", já a dificuldade do item é apresentada pelo parâmetro "c". Os cinco itens com maior dificuldade, em ordem crescente, foram 16, 15, 4, 2 e 24. Já os itens com menor dificuldade, também em ordem crescente, foram 34, 32, 33, 25 e 30. Esse parâmetro indica em qual região, na escala proposta, o item possui maior informação (Bortolotti et al., 2012).

0 grau de discriminação de um item determina a qualidade do item, isto é, quanto maior o valor de "a" maior o grau de discriminação na região de maior informação, consequentemente, melhor o 
Tabela 2. Itens que compõem o instrumento de medida.

\begin{tabular}{|c|c|}
\hline $\mathrm{N}^{\circ}$ & Descrição dos Itens \\
\hline 1 & Se utiliza algum conceito ou técnica estruturada para encontrar problemas e resolvê-los \\
\hline 2 & Se algo errado for identificado, a reação é procurar os motivos que ocasionaram o erro \\
\hline 3 & Se algo errado for identificado, a reação é tentar descobrir quem é o responsável pelo erro \\
\hline 4 & $\begin{array}{l}\text { É importante que o retorno sobre uma sugestão de melhoria seja realizado dentro de um tempo determinado pelos responsáveis pela } \\
\text { organização }\end{array}$ \\
\hline 5 & É importante que o retorno sobre alguma sugestão de melhoria seja realizado por meio de uma resposta clara e objetiva \\
\hline 6 & Se uma melhoria for iniciada é necessário que todas as atividades sejam realizadas até o final \\
\hline 7 & Se uma melhoria está sendo executada todas as pessoas do grupo devem participar das atividades \\
\hline 8 & Durante o desenvolvimento das melhorias, se utiliza algum formulário ou alguma técnica para dar apoio à execução das atividades \\
\hline 9 & É necessário que alguém estimule as pessoas do grupo para que as melhorias sejam desenvolvidas e aplicadas \\
\hline 10 & Durante o desenvolvimento das melhorias, é necessário que exista um monitoramento ou acompanhamento das atividades e resultados \\
\hline 11 & É fundamental que as melhorias sejam atreladas a metas e/ou objetivos do departamento ou da organização \\
\hline 12 & Se utilizam as estratégias ou objetivos do departamento para priorizar a execução das melhorias \\
\hline 13 & É importante que o gestor do setor aceite as melhorias propostas \\
\hline 14 & A participação do gestor do setor no desenvolvimento e aplicação de melhorias é importante \\
\hline 15 & É fundamental que o gestor do setor mostre liderança e comprometimento em relação ao processo de melhoria contínua \\
\hline 16 & 0 incentivo à realização de "melhorias piloto" sem cobrar resultados imediatos é necessário para motivar as pessoas \\
\hline 17 & Existe a necessidade de as pessoas receberem incentivos (brindes, dinheiro, etc.) para praticarem melhorias \\
\hline 18 & É importante realizar avaliações com a intenção de otimizar o processo de melhoria contínua \\
\hline 19 & Se uma melhoria for implementada, é importante que todos os envolvidos sejam treinados no novo método \\
\hline 20 & Se uma melhoria não está sendo executada de forma adequada, é necessário realizar um novo treinamento com os envolvidos \\
\hline 21 & Se uma melhoria é implantada, é necessário criar um procedimento para facilitar a aplicação do novo método \\
\hline 22 & Na organização, o processo de melhoria contínua deve ser percebido pelas pessoas como algo que faz parte da cultura da organização \\
\hline 23 & É importante que exista um comprometimento com o cumprimento dos horários e atividades do processo de melhoria contínua \\
\hline 24 & É necessário que exista orientação para a realização de melhorias entre setores na organização \\
\hline 25 & Ter o envolvimento de pessoas de vários níveis hierárquicos da organização nas melhorias mais elaboradas é fundamental \\
\hline 26 & Ter o apoio de clientes ou fornecedores no desenvolvimento de melhorias contribui para o processo \\
\hline 27 & É importante que os resultados das melhorias sejam continuamente medidos e monitorados pelos gestores da organização \\
\hline 28 & $\begin{array}{l}\text { Ter recursos suficientes (tempo, dinheiro, pessoal) para apoiar o desenvolvimento contínuo das melhorias na organização é fator } \\
\text { primordial para a continuidade de processo }\end{array}$ \\
\hline 29 & $\begin{array}{l}\text { Considerar opiniões externas ao grupo (gestores, técnicos de processo, etc.) é fundamental durante o desenvolvimento e aplicação das } \\
\text { atividades de melhoria }\end{array}$ \\
\hline 30 & Praticar o processo de melhoramento contínuo em melhorias já implementadas na empresa é relevante \\
\hline 31 & Se utiliza o procedimento operacional como base para o desenvolvimento e aplicação de melhorias \\
\hline 32 & Se utiliza indicadores de processo durante o desenvolvimento e aplicação das melhorias \\
\hline 33 & $\begin{array}{l}\text { É necessário que o conhecimento adquirido com o processo de melhoria contínua seja repassado para outras pessoas da organização } \\
\text { como forma de manter o processo ativo }\end{array}$ \\
\hline 34 & $\begin{array}{l}\text { Sempre que algo novo é aprendido é importante treinar as outras pessoas da organização para manter o aprendizado continuamente } \\
\text { renovado }\end{array}$ \\
\hline
\end{tabular}

Fonte: Elaborado pelos autores.

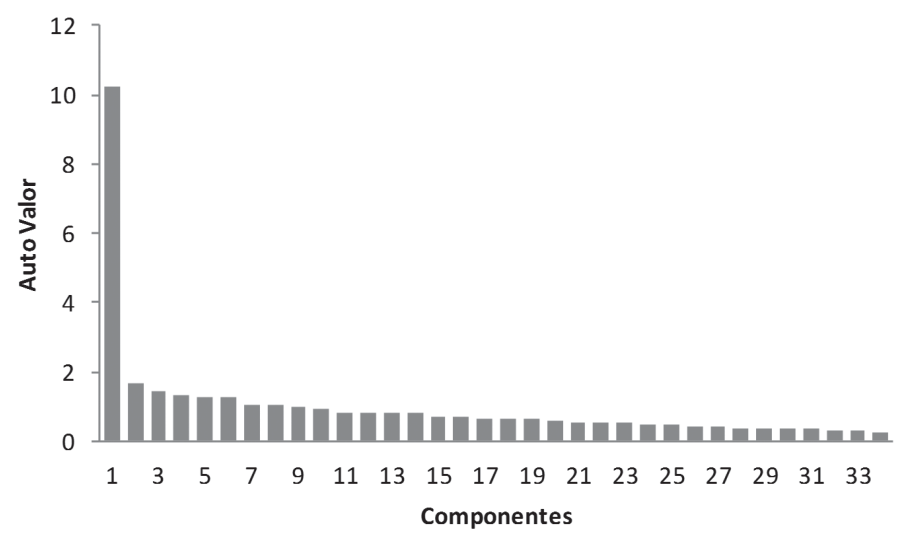

Figura 1. Representação gráfica da análise dos componentes principais. Fonte: Elaborado pelos autores. 
Tabela 3. Extração dos autovalores.

\begin{tabular}{ccccc}
\hline Fator & Autovalor & $\%$ & Autovalor Acum. & \% Acum. \\
\hline 1 & 10,2 & 30,0 & 10,2 & 30,0 \\
2 & 1,6 & 4,8 & 11,8 & 34,8 \\
3 & 1,4 & 4,3 & 13,3 & 39,1 \\
4 & 1,3 & 3,8 & 14,6 & 42,9 \\
5 & 1,3 & 3,8 & 15,9 & 46,7 \\
\hline
\end{tabular}

Fonte: Elaborado pelos autores.

Tabela 4. Estimativas dos parâmetros dos itens.

\begin{tabular}{|c|c|c|c|c|c|c|c|c|c|}
\hline Item & $a^{0}$ & $a^{1}$ & $c^{0}$ & $c^{\prime}$ & Item & $a^{0}$ & $a^{1}$ & $c^{0}$ & $c^{1}$ \\
\hline 1 & $-1,5$ & 1,96 & $-0,55$ & 0,28 & 18 & $-1,36$ & 1,5 & $-0,44$ & 0,11 \\
\hline 2 & $-2,12$ & 2,71 & $-0,82$ & 0,41 & 19 & $-0,88$ & 1,06 & $-0,35$ & 0,03 \\
\hline 3 & $-1,19$ & 1,5 & $-0,43$ & 0,34 & 20 & $-0,97$ & 1,1 & $-0,39$ & 0,03 \\
\hline 4 & $-1,07$ & 1,34 & $-0,43$ & 0,38 & 21 & $-1,1$ & 1,18 & $-0,5$ & 0,07 \\
\hline 5 & $-1,5$ & 1,72 & $-0,58$ & 0,2 & 22 & $-1,07$ & 1,19 & $-0,33$ & 0,05 \\
\hline 6 & $-1,45$ & 1,79 & $-0,61$ & 0,29 & 23 & $-0,93$ & 0,98 & $-0,3$ & 0,07 \\
\hline 7 & $-1,19$ & 1,56 & $-0,39$ & 0,29 & 24 & $-0,35$ & 0,51 & $-0,12$ & 0,42 \\
\hline 8 & $-1,33$ & 1,73 & $-0,34$ & 0,21 & 25 & -1 & 1,12 & $-0,35$ & $-0,05$ \\
\hline 9 & $-1,07$ & 1,41 & $-0,42$ & 0,32 & 26 & $-0,36$ & 0,51 & $-0,18$ & 0,32 \\
\hline 10 & $-1,17$ & 1,18 & $-0,25$ & 0,12 & 27 & $-0,92$ & 1,01 & $-0,43$ & $-0,02$ \\
\hline 11 & $-1,38$ & 1,45 & $-0,68$ & 0,13 & 28 & $-0,91$ & 0,97 & $-0,41$ & 0,04 \\
\hline 12 & $-1,13$ & 1,2 & $-0,43$ & 0,11 & 29 & $-0,54$ & 0,74 & $-0,26$ & 0,11 \\
\hline 13 & $-1,22$ & 1,33 & $-0,51$ & 0,08 & 30 & $-0,67$ & 0,78 & $-0,25$ & $-0,05$ \\
\hline 14 & $-1,14$ & 1,32 & $-0,41$ & 0,1 & 31 & $-0,8$ & 0,72 & $-0,27$ & $-0,03$ \\
\hline 15 & $-0,62$ & 0,8 & $-0,26$ & 0,36 & 32 & $-0,36$ & 0,37 & $-0,27$ & $-0,09$ \\
\hline 16 & $-0,7$ & 0,9 & $-0,27$ & 0,34 & 33 & $-0,46$ & 0,38 & $-0,13$ & $-0,08$ \\
\hline 17 & $-1,24$ & 1,38 & $-0,5$ & 0,07 & 34 & $-0,16$ & 0,17 & $-0,15$ & $-0,11$ \\
\hline
\end{tabular}

Fonte: Elaborado pelos autores.

item. Os cinco itens com maior qualidade, em ordem crescente, foram 2, 1, 6, 8 e 5. Já os cinco itens com menor qualidade, em ordem crescente, foram 24 , $26,33,32$ e 34 .

\section{Construção da escala de medida}

Essa etapa tem a finalidade de equiparar, ou seja, tornar comparável, os parâmetros dos itens e o nível dos colaboradores para a prática da Melhoria Contínua, colocando-os numa mesma métrica, isto é, numa escala comum, possibilitando assim, comparar itens e níveis (Bortolotti et al., 2012). Para efeitos práticos é possível transformar os valores dos parâmetros tanto dos itens como dos níveis em outros valores, mas mantendo as mesmas relações de ordem entre os pontos (Bortolotti et al., 2012). Nesse sentido, a escala para medir o nível dos colaboradores para a prática de Melhoria Contínua na organização foi desenvolvida com média 100 e desvio padrão 10.

Para construção da escala de medida, utilizaram-se como base os níveis âncora e quase âncora definidos por Beaton \& Allen (1992). E também, os conhecimentos desenvolvidos a partir de trabalhos de Bessant et al. (2001), Mesquita \& Alliprandini (2003) e Oprime \&
Lizarelli (2010), como forma de orientar a construção da escala. Na Figura 2 está representada a referida escala.

Seguindo a linha de raciocínio de Bessant et al. (2001), existem cinco níveis para a prática da Melhoria Contínua. Esses também podem ser utilizados para medir o nível dos colaboradores para a prática desse mesmo conceito e posteriormente avaliar o nível dessas práticas dentro da organização. Para construção da escala, somente alguns itens foram utilizados para caracterizar cada nível. lsso se deve ao fato de que alguns itens não continham a qualidade ou informação suficiente para representar o nível da escala ou ainda não estavam contidos nos critérios estabelecidos por Beaton \& Allen (1992), para instituir os itens âncoras ou quase âncoras. Assim sendo, os níveis que constituem a escala são:

N1 - Pré Melhoria Contínua: existe um interesse inicial pela prática motivado por uma crise, visita a outra organização ou participação em algum evento de melhoria, porém a prática é realizada de forma pontual.

N2 - Melhoria Contínua estruturada: existe um envolvimento com a prática que objetiva disseminar 
os conceitos da Melhoria Contínua por toda a organização por meio de um método adequado.

N3 - Melhoria Contínua orientada para metas: existe um compromisso com a prática da Melhoria Contínua e os resultados estratégicos esperados pela organização, ou seja, os colaboradores estão preocupados com a estratégia e o impacto das melhorias nos resultados.

N4 - Melhoria Contínua Proativa: os colaboradores têm autonomia e poder para gerenciar e dirigir seus próprios processos e suas iniciativas de melhorias. Neste nível, os colaboradores têm um senso de dono do processo muito elevado.

N5 - Plena capacidade para Melhoria Contínua: nesse nível os colaboradores, além de praticar, também exercem o papel de instrutores para a prática da Melhoria Contínua.

A Figura 3 mostra a frequência dos colaboradores distribuídos nos níveis da escala para a prática de Melhoria Contínua. Nessa figura, também foram colocados os percentuais de colaboradores que estão contidos nas escalas de medida.

Deste modo, ficou claro que 14\% dos colaboradores têm um score na escala de 104 (nível 1), ou seja, esses estão na condição de pré-melhoria, na qual o conceito já está sendo utilizado, motivado por visitas em outras empresas que já aplicam o conceito, ou por uma crise na organização, mas a implantação ainda é muito pontual.

No score 106 (nível 2), estão os colaboradores com um nível mais estruturado para a prática. Neste, existe um compromisso em construir um sistema de aplicação das melhorias por toda a empresa. Por conseguinte, no score 108 (nível 3), estão os colaboradores com nível para a prática orientado para metas. Existe um compromisso em relacionar as melhorias e a sua atuação operacional com os objetivos estratégicos da organização. Já, no score 110 (nível 4), estão os colaboradores com nível para a prática de melhorias proativas, ou seja, os problemas são resolvidos proativamente, buscando soluções de forma a gerenciar seus processos com eficiência.

Escala de Medida para o Nível da Prática de Melhoria Contínua

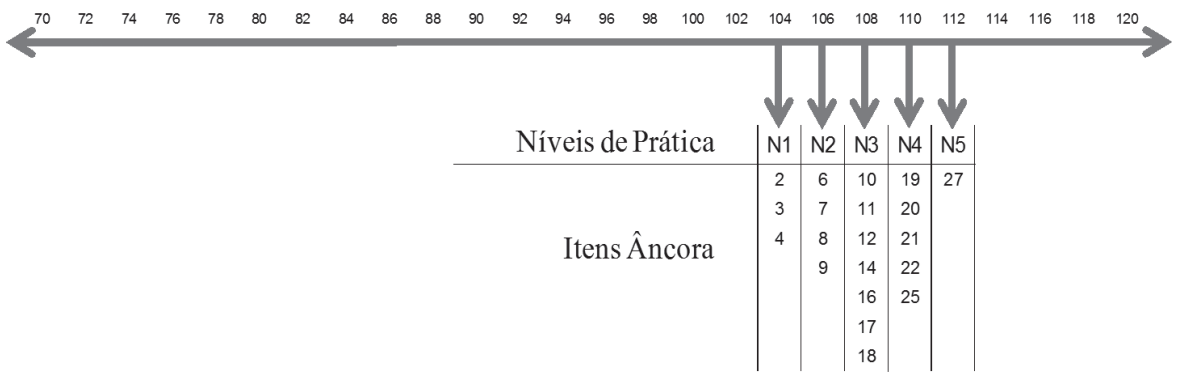

Figura 2. Escala de medida com os itens âncora e quase âncora. Fonte: Elaborado pelos autores.

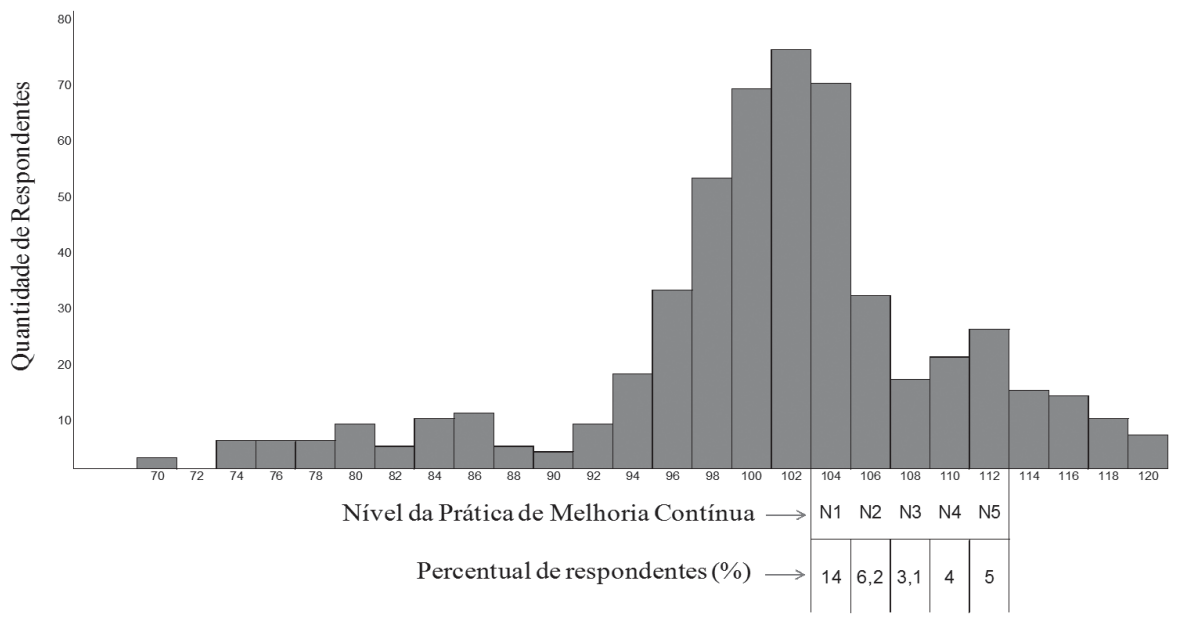

Figura 3. Histograma dos colaboradores nos diferentes níveis da escala de medida. Fonte: Elaborado pelos autores. 
E, finalmente, o score 112 (nível 5): nesse nível, os colaboradores aplicam o conceito de forma abrangente e autônoma, existe uma aquisição e compartilhamento de conhecimento muito grande entre os colaboradores dentro da organização.

\section{Considerações finais}

Com a finalidade de construir uma escala para medir o nível dos colaboradores para a prática da Melhoria Contínua na organização pesquisada, este trabalho aplicou o método estatístico da Teoria de Resposta ao ltem e, por meio do Modelo de Resposta Nominal, desenvolveu a referida escala. Como principal resultado, obteve-se uma equalização entre os itens aplicados e os scores dos indivíduos pesquisados. Consequentemente, viabilizou-se o desenvolvimento de uma escala de medida com diferentes níveis para a prática da Melhoria Contínua e a alocação dos itens do instrumento de avaliação nos níveis de prática estabelecidos.

Durante as análises dos parâmetros estimados pelo modelo de resposta nominal, foi verificado que somente alguns itens dentre os 34 utilizados poderiam constituir a escala para medir o nível para a prática da Melhoria Contínua. Assim sendo, foram retirados da escala os itens que não se enquadravam nos parâmetros para escolha de itens âncoras ou quase âncora, conforme Beaton \& Allen (1992), ou que tiveram seu grau de discriminação muito baixo. 0 percentual de itens desclassificados foi de $41 \%$. Outra ponderação refere-se à distribuição dos itens dentro das escalas. Dos 20 itens que constituem a escala, 15\% estão contidos no primeiro nível; 20\% estão no segundo nível; 35\% estão no terceiro nível; 25\% estão no quarto nível; e somente 5\% estão no último nível.

Além disso, fica claro na Figura 3 que a distribuição dos níveis está muito concentrada entre os pontos 104 e 114 da escala. Já a avaliação dos scores dos indivíduos mostrou que quase $70 \%$ dos respondentes estão acima ou abaixo dos níveis estabelecidos para a escala de medida. Se for observada a escala construída, os percentuais de indivíduos em cada nível ficaram distribuídos da seguinte maneira: $14 \%$ no primeiro nível; 6,2\% no segundo nível; 3,1\% no terceiro nível; 4\% no quarto nível; e 5\% no quinto nível.

Durante as análises dos parâmetros gerados e posteriormente na construção da escala de medida, ficou evidente que a quantidade de itens não foi suficiente para uma representação significativa ao longo da métrica calculada. lsso se deve por quatro pontos. Primeiro ponto: a amostra de respondentes não tinha uma distribuição equiparada entre os níveis da escala que se pretendia medir, mesmo sendo constituída por 519 indivíduos, a maioria está abaixo dos níveis da prática de melhoria esperado. Segundo ponto: a qualidade dos itens não foi suficiente para representar de forma qualitativa mais pontos na escala. E o terceiro ponto está relacionado aos poucos itens que passaram no processo de escolha dos itens âncoras e quase âncoras, dificultando assim a construção da escala. E ainda os itens escolhidos tinham seus scores muito próximos entre os níveis, gerando uma concentração nos níveis estabelecidos para a prática da melhoria contínua nas organizações.

Entretanto, o modelo proposto neste artigo serve de guia para ações de intervenção na medida do nível da prática da Melhoria Contínua na organização por meio da avaliação dos indivíduos que a compõem. Naturalmente que se faz necessário continuar os esforços de pesquisa no sentido de melhorar a escala proposta neste artigo por meio da construção de novos itens que possam representar melhor outros pontos na escala desenvolvida. Além disso, a aplicação desse instrumento em outras organizações e a calibração da escala de medida se fazem necessárias para que o instrumento possa ser utilizado de forma mais ampla por pesquisadores e organizações.

\section{Referências}

Araujo, E. A. C., Andrade, D. F., \& Bortolotti, S. L. V. (2009). Teoria da resposta ao item. Revista da Escola de Enfermagem da USP, 43, 1000-1008.

Beaton, A. E., \& Allen, N. L. (1992). Interpreting scales through scale anchoring. Journal of Educational Statistics, (17), 191-204.

Bessant, J., Caffyn, S., \& Gallagher, M. (2001). An evolutionary model of continuous improvement behavior. Technovation, 21(2), 67-77. http://dx.doi.org/10.1016/S0166-4972(00)00023-7.

Bessant, J., Caffyn, S., Gilbert, J., Harding, R., \& Webb, S. (1994). Rediscovering continous improvement. Technovation, 14(1), 17-29. http://dx.doi.org/10.1016/0166-4972(94)90067-1.

Bhuiyan, N., \& Baghel, A. (2005). An overview of continuous improvement: from the past to the present. Management Decision, 43(5), 761-771. http://dx.doi. org/10.1108/00251740510597761.

Bock, R. D. (1972). Estimating item parameters and latent ability when responses are scored in two or more nominal categories. Psychometrika, 37(1), 29-51. http://dx.doi. org/10.1007/BF02291411.

Bortolotti, S. L. V., Moreira Junior, F. J., Bornia, A. C., Sousa Júnior, A. F., \& Andrade, D. F. (2012). Avaliação do nível de satisfação de alunos de uma instituição de ensino superior: uma aplicação da teoria da resposta ao ltem. Gestão \& Produção, São Carlos, 19(2), 287-302. http:// dx.doi.org/10.1590/S0104-530X2012000200005.

Brannen, M. Y., Liker, J. K., \& Fruin, W. M. (1998). Recontextualisation and factory-factory knowledge transfer from Japan to the US: the case of NSK. In J. K. Liker, W. M. Fruin \& P. Adler (Eds.), Remade in America: transplating and transforming 
Japonese management systems (pp. 117-153). New York: Oxford Press.

Caffyn, S. (1999). Development of a continuous improvement self-assessment tool. International Journal of Operations \& Production Management, 19(11), 1138-1153 .

Chaves, N. M. D. (2005). Caderno de campo das equipes de melhoria contínua (1 ed.). Nova Lima: INDG Tecnologia e Serviços.

Cole, R. E. (2001). From continuous improvement to continuous inovation. Quality Management Journal Berkeley, 8(4), 1051-1056.

Filippini, R. (1997). Operations management research: some reflections on evolution, models and empirical studies in 0M. International Journal of Operations \& Production Management, 177-8), 655-670. http://dx.doi. org/10.1108/01443579710175583.

Gil, A. C. (1996). Como elaborar projetos de pesquisa (3 ed.) São Paulo: Atlas.

Harrison, A. (2000). Continuous improvement: the trade off between self-management and discipline. Integrated Manufacturing Systems, 11(3), 180-187. http://dx.doi. org/10.1108/09576060010320416.

Harrison, A., \& Storey, J. (1996). New wave manufacturing strategies: operational, organizational and human dimensions. International Journal of Operations \& Production Management, 16(2), 63-76. http://dx.doi. org/10.1108/01443579610109848.

Hernandez, J. M. C., \& Caldas, M. P. (2001). Resistência à mudança: uma revisão crítica. RAE - Revista de Administração de Empresas, 41(2), 31-45.

Jha, S., Noori, H., \& Michela, J. L. (1995). The dynamics of continuous improvement. International Journal of Quality Science, 1(1), 19-47.
Martins, P. G., \& Laugeni, F. P. (2006). Administração da produção. São Paulo: Saraiva.

Meijer, R. R. (2003). Test scoring. Applied Psychological Measurement, 271), 75-77. http://dx.doi.org/10.1177/014662102237799.

Mesquita, M., \& Alliprandini, D. H. (2003). Competências essenciais para melhoria contínua da produção: estudo de caso em empresas da indústria de autopeças. Gestão \& Produção, 10(1), 17-33. http://dx.doi.org/10.1590/ S0104-530X2003000100003.

Miguel, P., Martins, R., larozinski, A., Mello, C., Nakano, D., Batista, E., \& Vianna, W. (2009). Desafios para a metodologia de pesquisa em engenharia de produção. tópicos emergentes e desafios metodológicos em engenharia de produção: casos, experiências e proposições. Rio de Janeiro: ABEPRO.

Oprime, P. C., \& Lizarelli, F. L. (2010). Relação entre estrutura para melhoria contínua e desempenho e estrutura organizacional. Revista Produção, 10(2), 250-273.

Recht, R., \& Wilderom, C. (1998). Kaizen and culture: on the transferability of Japanese suggestion system. International Business Review, 7(1), 7-22. http://dx.doi.org/10.1016/ S0969-5931(97)00048-6.

Reckase, M. D. (1979). Unifactor latent trait models applied to multifactor tests: results and implications. Journal of Educational Statistics, (4), 207-230.

Richardson, R. J. (1989). Pesquisa social: métodos e técnicas. São Paulo: Atlas.

Ritzman, L. P., \& Krajewski, L. J. (2004). Administração da produção e operações. São Paulo: Prentice Hall.

Shiba, S. (1997). TQM: quatro revoluções na gestão da qualidade. Porto Alegre: Bookman.

\title{
Construction of a scale to measure the level of employee commitment in the practice of continuous improvement in an organization through the nominal response model of TRI
}

\begin{abstract}
This research provides a discussion of the construction of a scale to measure the level of employee commitment in the practice of continuous improvement within an organization. The development of this scale was enabled by the use of a measuring instrument consisting of 34 items guided by the concept of continuous improvement applied to a sample of 519 employees of the organization. The data were analyzed using statistical tools, such as the item response theory, specifically via the nominal response model. As a result, the equalization could be applied between the items, and the scores of individuals could be studied, allowing for the development of a metric with different levels for the practice of continuous improvement. The strategic outcomes are very important because the application of latent trait IRT has allowed for an assessment of the phenomenon under study with more accuracy and consistency, thus proving relevant for decision making within the research organization information.
\end{abstract}

\section{Keywords}

Continuous improvement. Industrial organization. Metric. 